\title{
Tratamiento de la Pre-eclampsia Grave y de la Eclampsia Estudio Prospectivo
}

\author{
Dres.: Alfonso Jubiz $\mathrm{H}^{*}{ }^{*}$ y William Mejía V.**
}

Centro de Investigaciones Médicas

Universidad de Antioquia. Facultad de Medicina.

\section{INTRODUCCION}

El tratamiento de la hipertensión que se desarrolla durante el embarazo, ha sufrido modificaciones a través del tiempo. Cada investigador defiende su plan terapéutico, algunos coinciden en recomendar un manejo agresivo, considerado éste como la terminación del embarazo $(1,2,4,14,13,15)$, y otros abogan por uno conservador, $(1,9)$. Sin embargo, se puede asegurar que el tratamiento de este síndrome es todavía empírico y que no existe uniformidad en los investigadores consultados (3, 6, 10).

La prevención juega un papel muy importante y es aquí donde la consulta prenatal precoz, frecuente $y$ de buena

* Profesor Titular Dpto. Obs-ginecologia. Fac. de Medicina U. de A.

** Profesor Titular. Fac. Nacional Salud Pública. U. de A.
Fundación Hospitalaria, San Vicente de Paúl. Medellín, diciembre 1 de 1981.

calidad, ha mostrado sus bondades. La diferencia se aprecia cuando se compara la morbi-mortalidad materna y perinatal (6) de los países muy desarrollados, con aquellos que se encuentran en vías de desarrollo.

El manejo terapéutico de este desorden es la consecuencia lógica de la causa que lo desencadena, la cual, hasta el presente continúa en la oscuridad. Diferentes drogas se han utilizado, comenzando por el sulfato de magnesio $y$ continuando con los diuréticos y diferentes hipotensores, los cuales, tienen distintos mecanismos de acción.

Se puede decir que el sulfato de magnesio ha resistido la prueba del tiempo porque entre 1906 y 1921 se publicaron los primeros artículos europeos mencionando su uso en la eclampsia. Sin embargo, es en 1925 cuando Lazard citado por Stenchever (12) lo utiliza en 17 casos de eclampsia y sólo tiene una muerte. Entre los hipotensores, se ha 
estudiado el clorhidrato de Hidralacina como dilatador arterioselectivo.

En el presente estudio se comparan los resultados obtenidos con el uso de estas drogas, solas o en combinación, para tratar 3 grupos de embarazadas con pre-ecjampsia grave y eclampsia.

\section{OBJETIVOS}

\section{General}

Comparar los resultados del tratamiento efectuado en tres grupos de pacientes con preeclampsia grave y eclampsia, utilizando solo o en combinación el Sulfato de Magnesio y la Hidralacina.

\section{Especificos}

1. Determinar la dosis promediò del Sulfato de Magnesio y de Hidralacina en tres grupos de embarazadas con preeclampsia grave y eclampsia, que consultaron al Departamento de Obstetricia y Ginecología de la Facultad de Medicina de la Universidad de Antioquia, entre el 10 . de abril de 1980 y el 30 de mayo de 1981.

2. Determinar el tiempo promedio que transcurre entre la aplicación de las drogas y el descenso de la tensión arterial diastólica a $90 \mathrm{mmHg}$.

3. Determinar el índice de Apgar al primer minuto y a los cinco, en los recién nacidos de las madres estudiadas.

4. Determinar el peso en gramos de los recién nacidos de las madres estudiadas.

5. Determinar los efectos secundarios leves, moderados y graves, producidos por las drogas en las pacientes tratadas.

6. Conocer el estado final de la salud materna en los tres grupos estudiados.
7. Conocer el resultado final de los recién nacidos de las madres estudiadas.

\section{Material y Métodos}

\section{Definición de Preeclampsia Grave:}

Es un síndrome que aparece durante la gestación, después de la vigésima cuarta semana, caracterizado por hipertensión de 160/110, edema y albuminuria.

\section{Definición de Eclampsia}

Es el mismo cuadro anterior pero acompañado de convulsiones o coma.

\section{Distribución de las pacientes}

De las pacientes con preeclampsia grave y eclampsia que consultaron al Departamento de Obstetricia y Ginecología entre el 1o. de abril de 1980 y el 30 de mayo de 1981, se seleccionaron al azar y en forma aleatoria $\mathbf{3 0}$ gestantes. Las pacientes fueron divididas en tres grupos de 10 y se les aplicó el tratamiento de la siguiente manera:

GRUPO I. Considerado como grupo control fue tratado con el sulfato demagnesio en la siguiente forma:

A un frasco de dextrosa al $5 \%$ en agua destilada, se agregaron 10 gramos de Sulfato de Magnesio al $20 \%$. Los primeros 5 gramos $(275 \mathrm{ml})$ de la solución se aplicaron en forma rápida durante unos 15 a 30 minutos ( 35 gotas $/ \mathrm{min}$ ), los restantes 5 gramos se administraron a la dosis de 1 gramo por hora (18 gotas/min). Durante el uso del sulfato se vigilaron el reflejo patelar, la respiración y la eliminación urinaria.

GRUPO II. Recibió el sulfato de magnesio a la misma dosis y en igual forma 
que el Grupo I. Además, se utilizó el Clorhidrato de Hidralacina a la dosis de 10 miligramos por vía venosa durante 15 minutos. Si la tensión arterial diastólica no descendió a $90 \mathrm{mmHg}$ se aplicó 5 miligramos cada 20 minutos, también por vía venosa, hasta alcanzar la cifra mencionada de tensión arterial diastólica.

GRUPO III. Este grupo sólo recibió el Clorhidrato de Hidralacina a la dosis de 20 mgs por vía venosa seguidos de 5 miligramos cada 20 minutos de acuerdo con la respuesta de la tensión arterial diastólica.

A todas las pacientes se les practicaron los siguientes exámenes complementarios:

Citoquímico de orina, estudio hematológico completo, estudio de coagulación, urea, creatinina, ácido úrico, albúmina en orina de 24 horas, depuración de creatinina y electrocardiograma.

No se incluyeron en este estudio pacientes con historia de úlcera péptica, hipertensión, enfermedad renal o vascular. Además, se excluyeron las pacientes mayores de 40 años por el peligro potencial del desprendimiento de un ateroma. Nunca se utilizó una dosis de Hidralacina superior a 400 mgs diarios para evitar artritis y la aparición de un lupus eritematoso.

\section{Estudio de la vitalidad fetal}

Para estudiar la vitalidad fetal se realizaron pruebas bio-eléctricas, analizando la respuesta de la frecuencia cardíaca fetal o los movimientos espontáneos del feto y pruebas con estímulos como el ejercicio y oxitocina. El estudio del l íquido amniótico para buscar la relación lecitina esfingomielina nos sirvió para determinar la madurez pulmonar.

\section{Sulfato de Magnesia}

El ion Magnesio es un catión intracelular y es el cuarto en el organismo humano. Su principal acción se ejerce sobre el sistema neuromuscular donde tiene efectos curarizantes por los siguientes mecanismos:

a. Disminución de la acetil-colina liberada en las terminaciones nerviosas.

b. Disminución de la acción de la acetilcolina en la placa terminal.

c. Depresión de la fibra muscular por disminución de su excitabilidad. A nivel periférico produce vasodilatación con descenso de la tensión arterial, efecto más o menos intenso según la dosis y la vía de administración (8).

\section{Clorhidrato de Hidralacina}

Se utiliza el monoclorhidrato de $\mathrm{Hi}$ dralacina, cuya fórmula es la siguiente:

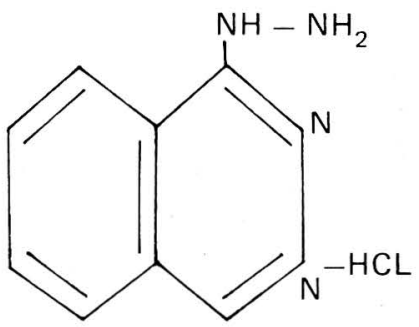

Su principal acción se ejerce en la musculatura lisa de los vasos provocando una vasodilatación que conduce a una disminución de la tensión arterial, especialmente de la diastólica. Su efecto puede ser prolongado en la pared muscular por la acción de los metabolitos (piruvato hidrazona y alfacetoglutarato hidrazona). Su uso en Obstetricia se extiende al manejo de la hipertensión inducida por el embarazo por acción sobre el flujo sanguíneo renal y uterino (5). 


\section{Resultados}

Al analizar la composición de los tres grupos de acuerdo con la tensión arterial, se encontró que son comparables.

Cuadro No. 1

\section{RELACION ENTRE LA EDAD} GESTACIONAL Y EL DIAGNOSTICO

\begin{tabular}{|l|ccc|ccc|c|}
\hline \multirow{2}{*}{$\begin{array}{c}\text { Edad } \\
\text { Gesta } \\
\text { cional }\end{array}$} & \multicolumn{2}{|c|}{$\begin{array}{c}\text { Pre-Eclampsia } \\
\text { Grave }\end{array}$} & \multicolumn{5}{|c|}{ Eclampsia } \\
\cline { 2 - 8 } & I & II & III & I & II & III & Total \\
\hline $25-27$ & & 1 & & & & & 1 \\
$28-30$ & - & 1 & 2 & - & 1 & - & 4 \\
$31-33$ & - & 1 & 1 & 1 & - & - & 3 \\
$34-36$ & & & & 1 & & & 1 \\
$37-39$ & 6 & 4 & 6 & 2 & 2 & 1 & 21 \\
\hline Total & 6 & 7 & 9 & 4 & 3 & 1 & 30 \\
\hline
\end{tabular}

El $70 \%$ de las pacientes ingresaron al Hospital con una gestación de término. La mayoría de ellas fueron remitidas de los Centros de Salud donde asistían a consulta prenatal. El $23.3 \%$ presentaron embarazos menores de 34 semanas.

Cuadro No. 2

FORMA DE TERMINACION DEL EMBARAZO

\begin{tabular}{|l|rrr|rrr|r|}
\hline & \multicolumn{2}{|c|}{ Pre-eclampsia Grave } & \multicolumn{4}{|c|}{ Eclampsia } \\
\cline { 2 - 8 } & I & II & III & I & II & III & Total \\
\cline { 2 - 8 } $\begin{array}{l}\text { Parto } \\
\text { Espontáneo }\end{array}$ & 1 & 3 & & 1 & & 5 \\
Fórceps & 2 & 1 & & 1 & 2 & 1 & 7 \\
Cesárea & 4 & 4 & 6 & 3 & 1 & & 18 \\
\hline Total & 6 & 6 & 9 & 4 & 4 & 1 & 30 \\
\hline
\end{tabular}

El $60 \%$ de los embarazos terminaron por cesárea, cifra reflejo del manejo agresivo que seguimos en las pacientes con preeclampsia grave $y$ eclampsia.
Cuadro No. 3

\section{PESO DE LOS RECIEN NACIDOS EN GRAMOS}

\begin{tabular}{|c|c|c|c|}
\hline Peso & $\begin{array}{c}\text { Pre-eclampsia } \\
\text { Grave }\end{array}$ & Eclampsia & Total \\
\hline $1.000-1.499$ & 5 & 2 & 7 \\
$1.500-.999$ & - & 1 & 1 \\
$2.000-2.499$ & 2 & 2 & 4 \\
$2.500-2.999$ & 10 & 2 & 12 \\
$3.000-3.499$ & 6 & 1 & 7 \\
\hline Total & 23 & 8 & $31^{*}$ \\
\hline
\end{tabular}

* Embarazo Gemelar

El $61.3 \%$ de los recién nacidos tuvieron un peso de 2.500 gramos o más, hecho relacionado con la edad gestacional, pues el $70 \%$ de las pacientes estudiadas ten ían gestación de término. Se incluye un embarazo gemelar, en una pre-eclampsia grave del grupo II, cuya gestación fue de 29 semanas.

\section{Cuadro No. 4}

RELACION ENTRE EL TRATAMIENTO Y EL INDICE DE APGAR AL PRIMER MINUTO

\begin{tabular}{|l|ccc|ccc|c|}
\hline & \multicolumn{3}{|c|}{$\begin{array}{c}\text { Pre-Eclampsia } \\
\text { Grave }\end{array}$} & \multicolumn{4}{|c|}{ Eclampsia } \\
\hline Apgar & I & II & III & I & II & III & Total \\
\hline R.N. muerto & & 2 & 2 & & & & 4 \\
$1-3$ & & 2 & & 3 & 2 & & $7^{*}$ \\
$4-6$ & 1 & 3 & 1 & 1 & & & $6^{*}$ \\
$7-9$ & 5 & 1 & 6 & & 1 & 1 & 14 \\
\hline Total & 6 & 8 & 9 & 4 & 3 & 1 & $31^{*}$ \\
\hline
\end{tabular}

\section{* Embarazo Gemelar}

El $45.1 \%$ de los recién nacidos se encontraron vigorosos al primer minuto; de éstos el $85.7 \%$ correspondió a preeclampsia grave. Sólo el $25 \%$ de los 
niños de las madres eclámpticas nacieron en buenas condiciones. El $13 \%$ de los fetos se encontraron muertos al ingreso, dos del grupo II y III.

Cuadro No. 5

RELACION ENTRE EL TRATAMIENTO Y EL INDICE DE APGAR A LOS CINCO MINUTOS

\begin{tabular}{|l|ccc|ccc|c|}
\hline & \multicolumn{2}{|c|}{$\begin{array}{c}\text { Pre-eclampsia } \\
\text { Grave }\end{array}$} & \multicolumn{4}{|c|}{ Eclampsia } \\
\hline Apgar & I & II & III & I & II & III & Total \\
\hline R.N.muerto & & 2 & 2 & & & & 4 \\
$1-3$ & & 1 & & 1 & & & 2 \\
$4-6$ & & 2 & & 1 & & & 3 \\
$7-9$ & 6 & 3 & 7 & 2 & 3 & 1 & 22 \\
\hline Total & 6 & 8 & 9 & 4 & 3 & 1 & $31^{*}$ \\
\hline
\end{tabular}

* Embarazo Gemelar

A los cinco minutos el $70.9 \%$ de los recién nacidos se encontraban en buenas condiciones. De éstos el $25.8 \%$ correspondieron a los grupos I y $\mathrm{III}$ respectivamente.

Cuadro No. 6

DISTRIBUCION DEL TIEMPO PROMEDIO EN MINUTOS Y SUS VARIABLES ENTRE LOS TRES GRUPOS ESTUDIADOS

\begin{tabular}{|l|r|r|r|r|r|r|r|}
\hline $\begin{array}{r}\text { Variable de } \\
\text { tiempo }\end{array}$ & $\bar{X}$ & D.S. & $\mathrm{O}_{1}$ & $\mathrm{O}_{2}$ & $\mathrm{O}_{3}$ & Max. & Min. \\
\hline $\begin{array}{l}\text { Sulfato } \\
\begin{array}{l}\text { Hidralacina } \\
\text { más sulfato }\end{array}\end{array}$ & 171.0 & 196.1 & 27.5 & 40 & 310 & 590 & 15 \\
Hidralacina & 32.5 & 245.0 & 20.0 & 90 & 420 & 720 & 15 \\
\hline
\end{tabular}

Al efectuar las pruebas estadísticas, se observa una diferencia significativa entre la Hidralacina (grupo III) y los otros dos grupos, con una $\mathrm{p}<0.05$. Por el contrario, no existe diferencia entre los grupos I y II porque $p>0.05$.

Desde el punto de vista estad ístico se muestra que la Hidralacina utilizada sola a una dosis no inferior de 20 mgs provoca una reducción de la tensión arterial diastólica más rápidamente y este efecto es más sostenido que con los otros tratamientos. Lo anterior se puede apreciar mejor en el gráfico No. 1.

En los gráficos 2, 3 y 4 se pueden observar los valores promedios de la tensión arterial en los 3 grupos.

Gráfica No. 2

DESCENSO PROMEDIO DE LA TENSION ARTERIAL GRUPO I MEDELLIN 1981

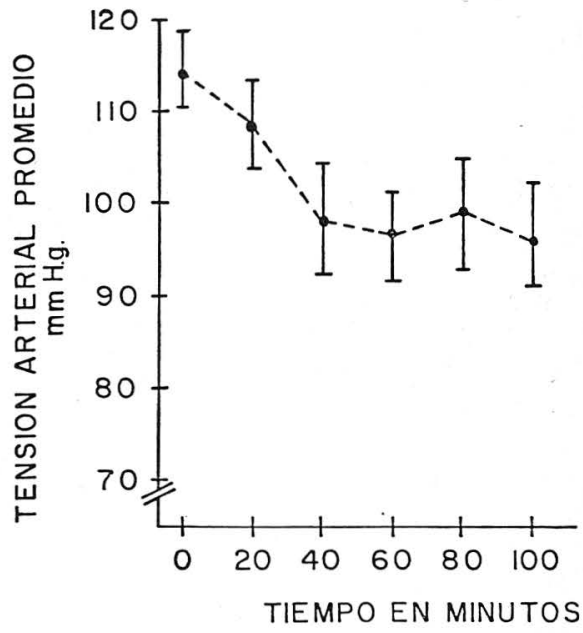

Dosis Promedio de las Drogas Utilizadas

La dosis promedio del Sulfato de Magnesio fue de 14.5 gramos, con una desviación estandar de 6.85 (grupo 1). Cuando se combinó con la Hidralacina fue de 12.2 grms, con una desviación 
Grafico No. 1

RELACION ENTRE EL TIEMPO EN MINUTOS Y EL DESCENSO

DE LA TENSION ARTERIAL DIASTOLICA SEGUN EL TRATAMIENTO

MEDELLIN 1981

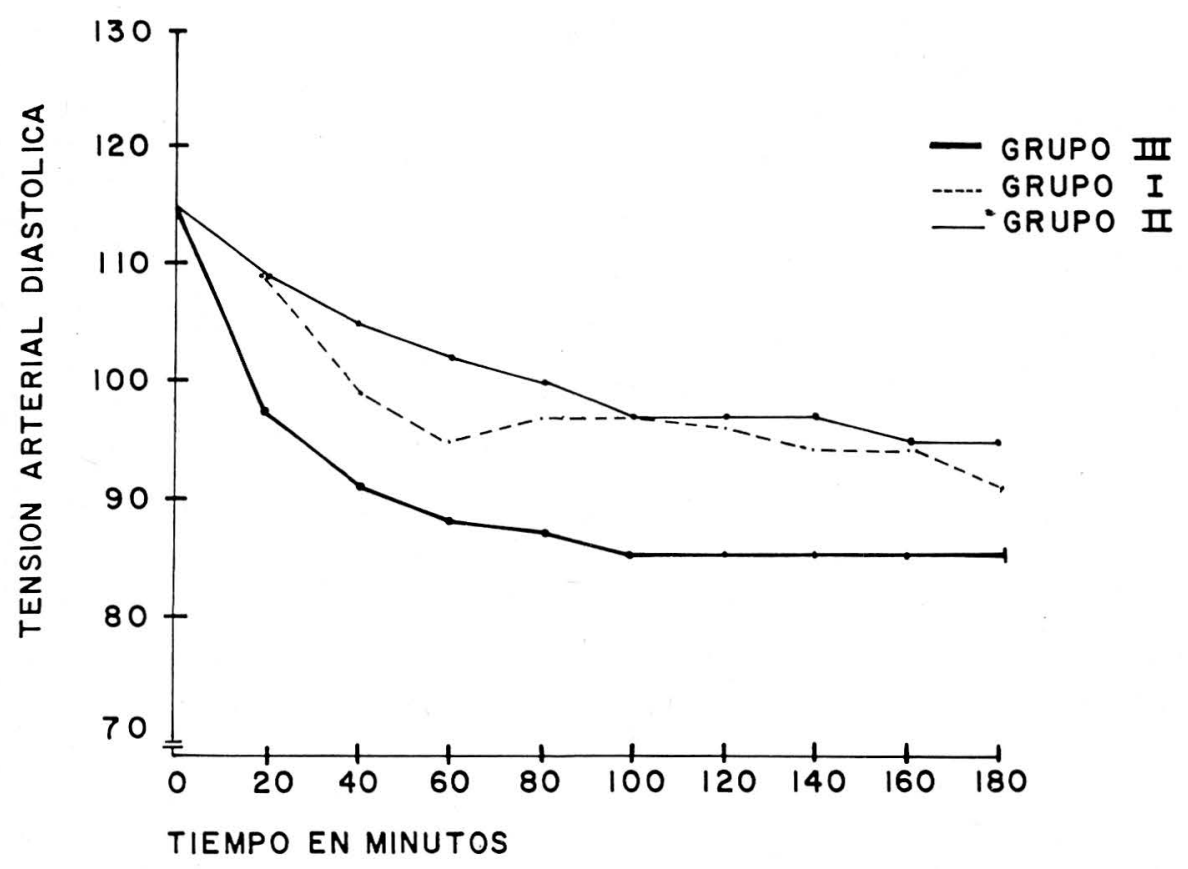

estandar de 5.6 gms; la Hidralacina promedio en el mismo grupo fue de 12.2 mgs, con igual desviación (grupo II).

Cuando la Hidralacina se utilizó sola para disminuir la tensión arterial diastólica, fue necesario una dosis promedio de 20.5 mgs, con una desviación estandar de 1.58 mgs.

\section{Estado final de los Recién Nacidos}

El total de niños muertos fue de 8, cuatro de los cuales ingresaron sin vida. La letalidad perinatal no corregida es de
$25.8 \%$ y la corregida de $13 \%$. El $74.2 \%$ de los neonatos abandonaron el Hospital en buenas condiciones.

\section{Morbi-Mortalidad Materna}

Una paciente con eclampsia incluída en el grupo I murió por hemorragia subaracnoidea $(3.33 \%)$. Otra eclampsia del grupo III presentó una hemorragia cerebral por ruptura de un aneurisma de la comunicante anterior, se colocó un clip de Scoville, la paciente sale con un síndrome frontal y hemiparesia izquierda moderada. 
Gráfica No. 3

DESCENSO PROMEDIO DE LA TENSION ARTERIAL GRUPO II MEDELLIN 1981

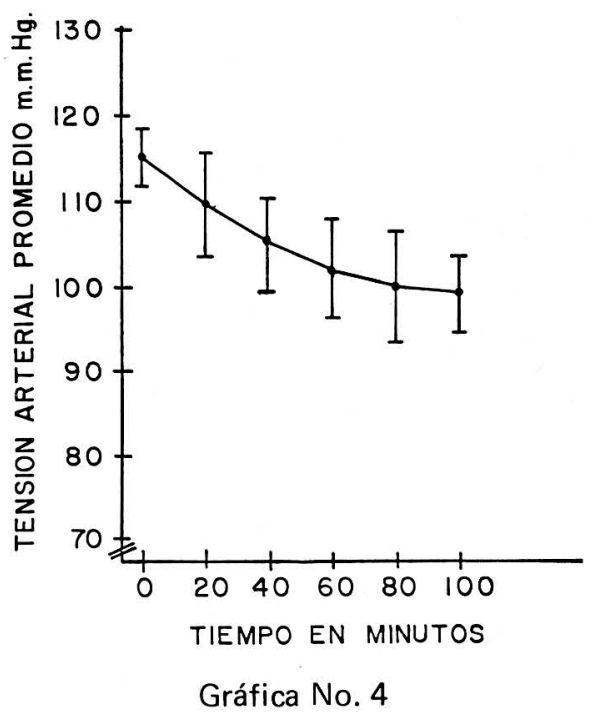

DESCENSO PROMEDIO DE LA TENSION ARTERIAL GRUPO III. MEDELLIN 1981

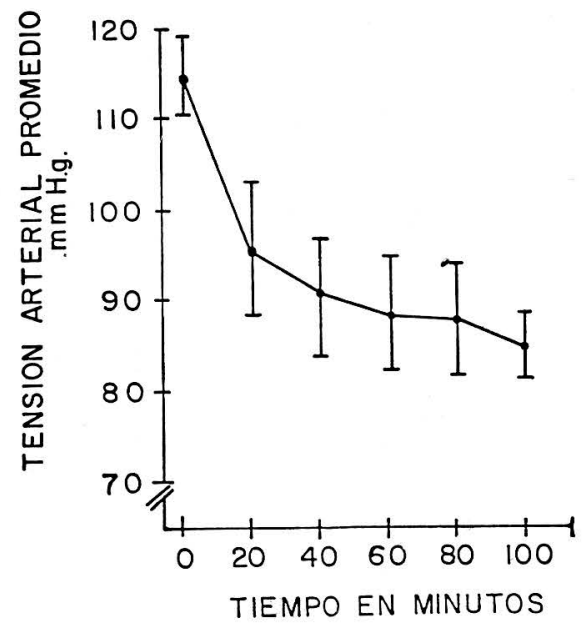

\section{Control Prenatal}

Hemos dejado ex-profeso esta variable para llamar la atención sobre la utilidad de la misma. El $53.4 \%$ de las pacientes no asistieron a ningún control prenatal. De las restantes $(46.6 \%)$ el promedio de consultas fue de 2.7 y en su mayoría tardíamente.

\section{Discusión}

Para iniciarla consideramos muy importante comenzar por el dato de la consulta prenatal: un poco más de la mitad de las pacientes habian asistido a control anteparto; creemos que el número y la calidad de los controles no cumplieron el objetivo de prevenir una de las complicaciones más graves de la embarazada. Es bien conocido el efecto que sobre la morbi-mortalidad materna y perinatal tiene la consulta prenatal en los países más desarrollados (6): en una revisión anterior recalcamos sobre este punto (7).

Zuspan desde 1965 (16) ha defendido el tratamiento de la hipertensión inducida por el embarazo, con Sulfato de Magnesio; recurre a la Hidralacina cuando la tensión arterial diastólica se encuentra en $110 \mathrm{mmHg}$. En el presente estudio el grupo tratado con Hidralacina a dosis de 20 mgs por vía venosa, presentó un efecto más rápido y sostenido sobre la diastólica, siguió luego el grupo çon Sulfato de Magnesio y por último, cuando se combinaron las dos drogas.

Consideramos que la preeclampsia y la eclampsia son emergencias obstétricas y que después de estabilizar la tensión arterial, la terminación del embarazo es la regla en la mayoría de los casos. Algunos autores consideran de importancia dividir el tratamiento de acuerdo con la edad gestacional (9). En el $60 \%$ de nuestras pacientes se practico cesárea, conducta semejante a la recomendada por Rizo y cols. (11). 


\section{Conclusiones}

1. Las pacientes del grupo III tuvieron un descenso más rápido y sostenido de la tensión arterial diastólica, $p<0.05$.

2. La dosis promedio del Sulfato de Magnesio fue de $14.5 \mathrm{gms}$ cuando se utilizó solo y de $12.2 \mathrm{gms}$ cuando se combinó con la Hidralacina.

3. La dosis promedio de Hidralacina fue de 20.5 mgms.

4. El $74.2 \%$ de los neonatos abandonaron el hospital en buenas condiciones.

5. La letalidad perinatal no corregida fue de $25.8 \%$, corregida de $13 \%$.

6. Se presentó una muerte materna $(3.33 \%)$.

\section{Resumen}

Se estudiaron tres grupos de embarazadas que -presentaron pre-eclampsia grave y eclampsia. En el grupo I se utilizó como tratamiento el Sulfato de Magnesio en el II, la Hidralacina más el Sulfato y en el grupo III la Hidralacina sola.

El $70 \%$ de las gestantes ingresaron con embarazo de término. El $26.7 \%$ presentaron eclampsia y las restantes $(73.3 \%)$ pre-eclampsia grave. El $60 \%$ de los embarazos terminaron por cesárea. Al efectuar las pruebas estadísticas se observa una diferencia significativa entre la Hidralacina (grupo III) y los otros dos grupos con una $\mathrm{p}<0.05$.

La Hidralacina utilizada a la dosis de $20 \mathrm{mgs}$ produce una disminución de la tensión arterial diastólica más rápida y este efecto es más sostenido.

\section{TREATMENT OF ADVANCED PRE-ECLAMPSIA AND ECLAMPSIA PROSPECTIVE STUDY}

\section{Summary}

Three groups of pregnant women, presenting both advanced pre-eclampsia and eclampsia. Group I was treated with magnesium sulphate: Group II, witn the sulphate plus hydralacine; and Group III with hydralacine alone.

$70 \%$ of the women studied were seen with a nine-month pregnancy. $26.7 \%$ had eclampsia and the remaining

\section{Bibliografía}

1. ANDERSEN, W.A. and HERBERT, Jr. G.M. Conservative management of preeclamptic and eclamptic patients: A reevaluation. Am. J. Obstet. Gynecol. 129: 260-66, 1977. women $(73.3 \%)$ had advanced preeclampsia.

In $60 \%$ of the cases a caesarian section was performed. Statistical analyses showed a significant difference between hydralacine (Group III) and the other two groups, with a $\mathrm{p} / 0.05$.

Hydralacine in a 20 mgs dose, produces a reduction of diastolic blood pressure in a shorter time and appears to be more sustained.

2. BASKETT, T.F. Active management of severe pre-eclampsia. Can Med. Ass. J. 109: 1. 209-11, Dec. 15, 1973.

3. BERKOWITZ, R.L. Anty-hypertensive drugs in the pregnant patient. Obstet. Gynecol. Surv. 35 (4): 191-203, 1980. 
4. FERRIS, F. Th. Toxemia and hypertension. In Burrow, G. and Ferris, F. The Medical complications during pregnancy. Philadelphia. Saunders. 1975, p. 92.

5. GOODMAN, A.G. GOODMAN, L.S. y GILMAN, A. Las bases farmacológicas de la terapeútica. B. Aires. Panamericana, 6a. Ed. (Inglés), 1981, p. 790-92.

6. HIBBARD, B.M. and ROSEN, M. The management of severe preeclampsia and eclampsia. Br. J. Anaesth 49: 3-9, 1977.

7. JUBIZ, H.A. MEDINA, B.S. y MEJIA, W. Morbi-Mortalidad materna y perinatal en pacientes con pre-eclampsia y eclampsia. Rev. Colomb. Obstet. Ginecol. 31 (5): 294-303, 1980.

8. LITTER, M. Farmacología Experimental y Clínica. B. Aires. El Ateneo 4a. Ed. 1969 p. 1020-23.

9. MARTIN, T.R. and TUPPER, W.R. The management of severe toxemia in patients at less than 36 weeks' gestation. Obstet. Gynecol. 54: 602, 1979.

10. PRITCHARD, J.A. Standardized treatment of 154 consecutive cases of eclamp- sia. Am. J. Obstet. Gynecol. 125: 74046, Jul. 15, 1976.

11. RIZO, S.J. et al. Conducta Obstétrica enla pre-eclampsia - eclampsia. En estado actual del tratamiento de la toxemia gravídica en el Hospital de la mujer. Publicación Técnica No. 9. Secretaría de Salud y Asistencia. México 1976. p. 29-39.

12. STENCHEVER, M.A. Magnesium, its relations hip to Obstetrics. Obstet. Gynecol. Surv. 16: 297, 1961.

13. SULLIVAN, J.M. Hypertension in pregnancy. Clin. Perinatol. 1 (2): 369-384, Sept. 1974.

14. TEJANI, S.N. et al. Modern management of hypertension disorders of pregnancy. Obstet. Gynecol 51 (6): 648-53, June. 1978.

15. WELT, S.I. and CRENSHAW, Jr. C. Concurrent hypertension and pregnancy Clin. Gynecol. 21 (3): 619, Sept. 1978.

16. ZUSPAN, F.P. and WARD, C.M. Improved fetal salvage in eclampsia. Obstet. Gynecol (26 (6): 893-897, Dec. 1965. 